\title{
Transcriptome analysis provides a blueprint of coral egg and sperm functions
}

\author{
Julia Van Etten ${ }^{1}$, Alexander Shumaker ${ }^{2}$, Tali Mass ${ }^{3}$, Hollie Putnam ${ }^{4}$, Debashish Bhattacharya ${ }^{\text {Corresp. } 5}$ \\ ${ }^{1}$ Graduate Program in Ecology and Evolution, Rutgers, The State University of New Jersey, New Brunswick, New Jersey, United States \\ 2 Microbial Biology Graduate Program, Rutgers, The State University of New Jersey, New Brunswick, New Jersey, United States \\ 3 Department of Marine Biology, University of Haifa, Haifa, Israel \\ 4 Department of Biological Sciences, University of Rhode Island, Kingston, Rhode Island, United States \\ 5 Department of Biochemistry and Microbiology, Rutgers, The State University of New Jersey, New Brunswick, New Jersey, United States \\ Corresponding Author: Debashish Bhattacharya \\ Email address: d.bhattacharya@rutgers.edu
}

Background. Reproductive biology and the evolutionary constraints acting on dispersal stages are poorly understood in many stony coral species. A key piece of missing information is egg and sperm gene expression. This is critical for broadcast spawning corals, such as our model, the Hawaiian species Montipora capitata, because eggs and sperm are exposed to environmental stressors during dispersal. Furthermore, parental effects such as transcriptome investment may provide a means for cross- or transgenerational plasticity and be apparent in egg and sperm transcriptome data.

Methods. Here, we analyzed $M$. capitata egg and sperm transcriptomic data to address three questions: (1) Which pathways and functions are actively transcribed in these gametes? (2) How does sperm and egg gene expression differ from adult tissues? (3) Does gene expression differ between these gametes?

Results. We show that egg and sperm appear to have surprisingly similar levels of gene expression and overlapping functional enrichment patterns. These results may reflect similar environmental constraints faced by these motile gametes. We find significant differences in differential expression of egg vs. adult and sperm vs. adult RNA-seq data, in contrast to very few examples of differential expression when comparing egg vs. sperm transcriptomes. Lastly, using gene ontology and KEGG orthology data we show that both egg and sperm have markedly repressed transcription and translation machinery compared to the adult, suggesting a dependence on parental transcripts. We speculate that cell motility and calcium ion binding genes may be involved in gamete to gamete recognition in the water column and thus, fertilization. 


\section{Transcriptome analysis provides a blueprint of coral egg and sperm}

\section{2 functions}

3

4 Julia Van Etten ${ }^{1}$, Alexander K. Shumaker², Tali Mass ${ }^{3}$, Hollie M. Putnam4, Debashish

5 Bhattacharya ${ }^{5}$

$7{ }^{1}$ Graduate Program in Ecology and Evolution, Rutgers, The State University of New Jersey,

8 New Brunswick, New Jersey 08901, United States

$9{ }^{2}$ Microbial Biology Graduate Program, Rutgers, The State University of New Jersey, New

10 Brunswick, New Jersey 08901, United States

$11{ }^{3}$ Department of Marine biology, Leon H, Charney school of Marine Sciences University of

12 Haifa, Israel

$13{ }^{4}$ Department of Biological Sciences, University of Rhode Island, Kingston, Rhode Island 02881,

14 United States

$15{ }^{5}$ Department of Biochemistry and Microbiology, Rutgers, The State University of New Jersey,

16 New Brunswick, New Jersey 08901, United States

Corresponding author:

Debashish Bhattacharya

59 Dudley Road, Foran Hall 102, New Brunswick, New Jersey 08901 USA

Email address: $\underline{\text { d.bhattacharya@,rutgers.edu }}$

\section{Abstract}

25 Background. Reproductive biology and the evolutionary constraints acting on dispersal stages are poorly understood in many stony coral species. A key piece of missing information is egg and sperm gene expression. This is critical for broadcast spawning corals, such as our model, the Hawaiian species Montipora capitata, because eggs and sperm are exposed to environmental stressors during dispersal. Furthermore, parental effects such as transcriptome investment may 
30 provide a means for cross- or trans-generational plasticity and be apparent in egg and sperm

31 transcriptome data.

32

33 Methods. Here, we analyzed $M$. capitata egg and sperm transcriptomic data to address three questions: (1) Which pathways and functions are actively transcribed in these gametes? (2) How does sperm and egg gene expression differ from adult tissues? (3) Does gene expression differ between these gametes?

Results. We show that egg and sperm appear to have surprisingly similar levels of gene expression and overlapping functional enrichment patterns. These results may reflect similar environmental constraints faced by these motile gametes. We find significant differences in differential expression of egg vs. adult and sperm vs. adult RNA-seq data, in contrast to very few examples of differential expression when comparing egg vs. sperm transcriptomes. Lastly, using gene ontology and KEGG orthology data we show that both egg and sperm have markedly repressed transcription and translation machinery compared to the adult, suggesting a dependence on parental transcripts. We speculate that cell motility and calcium ion binding genes may be involved in gamete to gamete recognition in the water column and thus, fertilization.

\section{Introduction}

Reef-building corals and their photosynthetic dinoflagellate endosymbionts form the structural foundation of complex ecosystems, supporting approximately $25 \%$ of marine biodiversity and protecting coastlines from damaging wave energy (Hughes et al. 2017). The rice coral Montipora capitata is a dominant reef-builder in the Hawaiian Archipelago that is of interest because of its demonstrated resilience throughout ocean warming events that can lead to loss of algal symbionts (termed "bleaching") and mortality (Grottoli et al. 2006). M. capitata has a relatively large genome (ca. $886 \mathrm{Mbp}$ in size) due primarily to high repeat and transposable element content. This genome expansion likely resulted from genetic drift due to small effective population size in the isolated Hawaiian island chain (Shumaker et al. 2019). These features, along with its relatively high tolerance to heat stress and ocean acidification (Gibbin et al. 2015), 
61 studying the emergence of locally adaptive traits and physiological responses of corals to 62 environmental change.

M. capitata is a broadcast-spawning hermaphrodite that annually releases bundles of sperm and eggs into the water column at roughly three summer intervals, according to the lunar cycle. Gametes undergo sexual fusion and produce larvae that settle, metamorphose, and develop into the meta-organisms that ultimately build reefs (Padilla-Gamiño et al. 2011; Padilla-Gamiño and Gates 2012). Broadcast-spawning is the most common form of reproduction within Scleractinia (stony corals) and is highly conserved within this order (Baird et al. 2009; PadillaGamiño et al. 2011). Unlike brooding corals, M. capitata gametes experience direct and prolonged exposure to the marine environment (within bundles, and thereafter upon release at the sea surface) during the several days of development and $\sim$ weeks of pelagic larval duration (Concepcion et al. 2012). Gamete survival in the water column is a period of a few hours during which they must abide environmental challenges such as predation, infection by microbial pathogens, and fluctuations in temperature and $\mathrm{pH}$, before taking part in the fertilization process (Baird et al. 2009) and larval recruitment. Despite the significance of gamete survival in the marine environment and its effect on overall recruitment potential in broadcast-spawning corals, few studies have focused primarily on egg and sperm cells and only one recent study has investigated gamete transcriptomics in the context of gametogenesis (Chiu et al. 2020). Moreover, investigation of the potential impacts of bleaching on reproduction has not shown significant differences in egg quality or spawning potential between bleached and non-bleached M. capitata (Cox 2007), despite the potential for parental investment in egg algal symbiont populations through vertical transmission. This reproductive resilience may be explained in part by an increase in adult heterotrophy, offsetting energetic losses due to bleaching (Grottoli et al. 2006), or the timing of gametogenesis relative to the thermal stress. However, bleaching events have been shown to negatively impact the degree of spawning for years after their occurrence, even in corals that did not show visible bleaching during the initial event (Levitan et al. 2014), as well as some gamete characteristics including reduced egg volume, longer time to first cleavage, and sperm motility (Hagedorn et al. 2016). Sperm play an equally important role in determining spawning success, yet little is known about sperm gene expression. Sperm DNA has been used to generate genome assemblies due to its high seasonal abundance and symbiont-free state, 
91 therefore data are readily obtainable for sperm-specific studies (e.g., Putnam et al. 2017;

92 Shumaker et al. 2019).

93 Here, we analyzed egg and sperm RNA-seq data generated from M. capitata colonies

94 located on fringing reefs near the Hawai'i Institute of Marine Biology (HIMB) in O`ahu (for

95 details on related analyses, see Putnam et al. 2017). Six individual adult colonies were sampled

96 to obtain three individual egg RNA-seq libraries and three individual sperm RNA-seq libraries

97 (both from ambient conditions) were sequenced on the Illumina platform using the Illumina

98 TruSeq RNA Library Preparation Kit v2. The egg data are publicly available under NCBI

99 BioProject PRJNA616341 (SAMN14486762, SAMN14486763, SAMN14486764) and the

100 sperm data are publicly available under NCBI BioProject PRJNA339779. We determined which

101 genes are expressed in coral egg and sperm cells and studied their putative functions. We

102 compared cDNA data from eggs and sperm to that of RNA isolated from tissues of three

103 individual un-stressed adults generated in a previous project (Shumaker et al. 2019) to determine

104 the degree of differential gene expression (DEG) between gametic and adult tissues. We then

105 compared gene expression of egg and sperm to identify functions that are shared or distinguish

106 these gametes. Finally, we used the expression data to highlight pathways represented by the

107 most differentially expressed genes in egg and sperm and compared gamete-specific functions

108 that account for various known physiological processes in M. capitata.

109

\section{Materials \& Methods}

\section{Sperm sample collection and sequencing library preparation}

112 M. capitata sperm were collected from the fringing reefs on the west side of Moku o Lo'e and

113 RNA extracted as described in Putnam et al. (2017). Three individual RNA-seq libraries were

114 generated using $200 \mathrm{ng}$ of the total RNA from each sample using the Illumina TruSeq RNA

115 Library Preparation Kit v2. The libraries were combined in equimolar concentrations and run on

116 a single Illumina MiSeq flowcell using the Illumina MiSeq Reagent Kit v3 (150 cycles, single-

117 end). The genomic and transcriptomic data created for this project are available under NCBI

118 BioProject PRJNA339779 and the three libraries were pooled together and can be found in

119 BioSample: SAMN05607941; Sample name: Mcap_nonBleach_RNA; SRA: SRS1632867.

120

121 Transcriptomic and genomic data analysis of sperm 
122 After trimming for quality (parameters shown in Fig. S1) using the CLC Genomics Workbench

123 8.5.1 (Qiagen, Hilden, Germany), the combined sperm RNA-seq data yielded 29,815,942 high

124 quality reads for assembly. The M. capitata genome (Shumaker et al. 2019) and structural

125 annotations used for the mappings are available at http://cyanophora.rutgers.edu/montipora/. The

126 libraries were individually mapped to the reference genome using CLC Genomics Workbench

127 (count data can be found in Table S1). Only "unique exon reads" counts (i.e., the number of

128 reads that match uniquely to exons, including across exon-exon junctions) were used for

129 downstream analyses.

130

131 Egg sample collection

132 In the June 2018 (6/13/18) spawning period, egg-sperm bundles were collected from ambient 133 conditions in the field on the fringing reefs at the HIMB under Special Activity Permit 2018-50

134 from the Hawai'i Department of Aquatic Resources. Bundles were brought back to the lab and

135 buoyant eggs were separated from the dense sperm after the bundles broke up. The eggs were 136 subsequently rinsed 3 times with $0.2 \mu \mathrm{m}$ filtered seawater and then following the removal of the

137 water, replicate tubes of eggs were snap frozen in liquid nitrogen and stored at $-80^{\circ} \mathrm{C}$.

138

\section{Egg RNA extraction and sequencing library preparation}

140 RNA was extracted using a dual DNA/RNA extraction method (Quick-DNA/RNA Miniprep

141 Plus Kit, Cat\# D7003). A total of 300 $\mu 1$ of Zymo DNA/RNA shield, $30 \mu 1$ of PK digestion buffer

142 was added to each sample tube, followed by the addition of $5 \mu 1$ Proteinase K. Next, samples

143 were vortexed, spun down, and placed in a Thermomixer for $1 \mathrm{~h}$ at $55^{\circ} \mathrm{C}$, shaking at $1100 \mathrm{rpm}$.

144 After heating, samples were and centrifuged at $2200 \mathrm{rcf}$ for one minute to separate any remaining

145 solids. The supernatant was transferred to a new microfuge tube and equal volume of DNA/RNA

146 Lysis Buffer was added and mixed. All liquid was transferred to a new Spin-Away filter column

147 with collection tube and spun at 16,000 rcf for $30 \mathrm{sec}$. The flow through containing the RNA

148 fraction was added to a $1.5 \mathrm{~mL}$ tube with an equal volume of $100 \% \mathrm{EtOH}$ was and tubes were 149 vortexed and spun down. A total of $700 \mu \mathrm{l}$ of the resulting mixture was added to the RNA spin

150 columns where it was centrifuged at 16,000 $\mathrm{rcf}(\mathrm{g})$ for $30 \mathrm{sec}$ and the flow through (Zymo kit

151 waste) was subsequently discarded. This step was repeated and then $400 \mu 1$ DNA/ RNA Wash

152 Buffer was gently added to each RNA column. The samples were centrifuged at 16,000 $\mathrm{rcf}(\mathrm{g})$ 
153 for $30 \mathrm{sec}$ and the flow through (Zymo kit waste) was discarded. Next, $80 \mu 1$ DNase I treatment 154 master mix (75 $\mu 1$ DNA Digestion buffer $\mathrm{x}$ \# of samples, $5 \mu 1$ DNase I x \# of samples) was added 155 directly to the filter of the RNA columns and incubated at room temp for $15 \mathrm{~min}$. Then, $400 \mu \mathrm{l}$ of

156 the DNA/ RNA Prep Buffer was gently added to each column and the mixture was centrifuged at $15716,000 \mathrm{rcf}$ (g) for $30 \mathrm{sec}$ and the flow through (Zymo kit waste) was discarded. The columns 158 containing the bound RNA were then transferred to new $1.5 \mathrm{~mL}$ microcentrifuge tubes and $50 \mu 1$ 159 of warmed DNase/RNase free water was added to each RNA column by dripping slowly directly 160 on the filter. The samples were incubated at room temperature for $5 \mathrm{~min}$ and centrifuged at $16116,000 \mathrm{rcf}(\mathrm{g})$ for $30 \mathrm{sec}$. This step was repeated to obtain a final elution volume of $100 \mu 1$, and 162 tubes were stored at $-80^{\circ} \mathrm{C}$. The cDNA libraries were prepared and sequenced by Genewiz using 163 standard Illumina strand-specific RNA-seq preparation with poly-A selection and then sequenced 164 with the HiSeq instrument using 2 x 150 bp reagents with $\sim 15 \mathrm{M}$ raw paired-end reads per 165 sample.

\section{Transcriptomic and genomic data analysis of eggs}

168 After trimming for quality (parameters shown in Fig. S1) using the CLC Genomics Workbench 169 8.5.1 (Qiagen, Hilden, Germany), the combined egg RNA-seq data yielded 125,054,213 high 170 quality paired reads for assembly. The M. capitata genome (Shumaker et al. 2019) and structural 171 annotations used for the mappings are available at http://cyanophora.rutgers.edu/montipora/. The 172 libraries were individually mapped to the reference genome using CLC Genomics Workbench 173 8.5.1 (QIAGEN, Aarhus Denmark) (count data can be found in Table S2). Only "unique exon 174 read" counts were used for downstream analyses.

\section{Functional Analysis}

Read count normalization

178 Read counts across the three egg and three sperm libraries, as well as three adult ambient 179 condition RNA-seq runs from Shumaker et al. (2019) used in DEG analysis, were normalized to 180 transcripts per million (TPM). The number of genes expressed at TPM thresholds between 0 and 181200 (in increasing increments of 10) were tabulated and used to determine the proportion of the 182 gene inventory expressed at each threshold. These values were converted to percent of total 183 genes in the genome, and that percentage was plotted on the y-axis vs. the incremental thresholds 
184 on the $\mathrm{x}$-axis. Based on the graphs for each of the three datasets (egg, sperm, adult), there is a

185 steep drop off in the percentage of genes around 100 TPM in both the egg and sperm data, where

$186>100$ TPM represents $12.05 \%(7,620 / 63,227)$ and $12.31 \%(7,783 / 63,227)$ of the total genes,

187 respectively. In the adult read counts, $\sim 12 \%$ of all genes corresponds to 60 TPM, however,

188 because this group had many more expressed genes (TPM $>0,58.69 \%$ ) compared to the egg and

189 sperm (31.98\% and 30.27\% respectively), the drop-off in percentages of genes above the TPM

190 threshold increments occurs very early in this dataset. Because the adult data are not relevant to

191 this particular part of the analysis, we chose 100 TPM to be a reasonable threshold for moving

192 forward with the egg and sperm data because although arbitrary, this threshold reflects

193 consistency in proportion of the genes represented across these datasets. Normalized read counts

194 and accompanying graphs are provided in the Supplementary Spreadsheet (Tab 2).

195

196 Gene expression in egg and sperm based on gene ontology

197 To determine which biological, cellular, and molecular functions are most prevalent across the

198 combined egg and combined sperm RNA-seq data, we used the 100 TPM threshold to generate a

199 list of "expressed" genes in both the egg and sperm which consisted of 7,620 and 7,783 genes

200 respectively. The Blast2GO software was used to map GO terms to the $M$. capitata gene

201 inventory and to test for enrichment among the sets of “expressed” genes using Fisher's Exact

202 Test (Götz et al. 2008), using the gene inventory as the reference set.

203

204 Using GO terms to organize KEGG pathway data

205 These sets of "expressed genes" were assigned KEGG Orthology (KO) terms using the

206 KofamScan software (Aramaki et al. 2019), yielding 4,078 genes in the "egg" set with at least

207 one KO term and 3,146 genes in the "sperm" set with at least one KO term. Each set of KOs

208 (egg, sperm) was mapped onto well-studied biological pathways using the "Reconstruct

209 Pathway" tool (Kanehisa 2017). Next, all KEGG pathways associated with each KO (excluding

210 human-specific pathways) were retrieved and cross-referenced with GO terms of the category

211 "cellular component". This allowed for assignment of each KO term and its associated

212 pathway(s) to a putative location within the cell. The exhaustive list of all KEGG pathway-GO

213 pairs is included in the supplemental spreadsheet (Tabs 4 and 6) and the most common terms

214 relevant to each cellular component were selected as the functional pathways to display in our 
215 analysis. This analysis was done to make an overall assessment of which metabolic processes are

216 most frequently expressed in egg and sperm cells based on the normalized read counts for RNA-

217 seq transcript data, and to identify if there are any major differences in overall functionality

218 between each gamete type that can be further investigated.

219

220

\section{Differential Gene Expression Analysis}

221

DEG analysis was conducted using the DESeq2 package (Love et al. 2014) using the raw counts

222 as input (parameters shown in Fig. S2). The count matrices and column data used as input can be found in supplementary spreadsheet (Tabs 8, 9, and 12). Differentially expressed genes were identified in contrasts between egg and adult samples and sperm and adult samples, as well as between egg and sperm samples. Egg replicates corresponding to individual RNA-seq runs are labeled E1, E2, E3, sperm samples are labeled Ub2, Ub3, Ub4, and ambient control treatment adult samples from Shumaker et al. (2019) are labeled W1, W5, and W7 in all related tables and 228 figures.

\section{Results}

231 Functional Analysis

232 Fisher's exact test revealed similar functional enrichments among the "expressed" gene sets 233 (TPM > 100) in both gamete types. Among the Biological Process and Molecular Function GO

234 terms enriched in the "expressed" genes in the egg samples, the top ten are 'ATP binding', 'GTP 235 binding', 'GTPase activity', 'structural constituent of ribosome', 'chromatin binding', 236 'microtubule motor activity', 'negative regulation of transcription by RNA polymerase II', 237 'protein polyubiquitination', 'translation initiation factor activity', and 'ubiquitin ligase activity'.

238 The top ten enriched GO terms for sperm “expressed” vs. all genes are 'ATP binding', 'GTP 239 binding', 'GTPase activity', 'structural constituent of ribosome', 'chromatin binding',

240 'microtubule binding', 'endonuclease activity', 'microtubule motor activity', 'negative

241 regulation of transcription by RNA polymerase II', and 'protein polyubiquitylation'. The

242 complete list of the GO terms for each of these enrichment profiles and their dataset of origin are 243 presented in Table 1. These GO lists, their prevalence in the M. capitata genome, and degree of 244 enrichment are shown in Figure S3. All GO term enrichments beyond the top 30 shown here 245 were minor and not reported. 


\section{Using GO terms to organize KEGG pathway data}

248 After indexing KO terms by their corresponding Cellular Component GO terms, we determined

249 how much of each transcriptome (TPM of all genes assigned to each GO term divided by total

250 TPM of all genes in each "expressed" dataset) is associated with each cellular component and

251 found this metric to be very similar between the egg and sperm datasets (see Figure 1). To then

252 determine the underlying functional pathways associated with these locations within the cell, the

253 top KEGG pathways for selected parts of the cell were identified, excluding those that are

254 human/ mammal-specific or disease-related. Each KO term was counted for each part of the cell

255 and the most frequent terms were included in Figure 2. The complete list of all of the raw data

256 used in this analysis can be found in the supplemental spreadsheet (Tabs 5 and 7). These results

257 further support the Biological Process and Molecular Function GO data shown above by

258 confirming the similarity between the egg and sperm with respect to which pathways are most

259 prominently expressed and where they are active within the cell. The functional implications of

260 these differences will be discussed below.

261

\section{Differential gene expression analysis}

263 Gamete vs. adult

264 From a broader perspective, and perhaps surprisingly, $M$. capitata egg and sperm appear to have

265 similar patterns of gene expression and share functional enrichment patterns. However, in order

266 to gain a more in-depth understanding of how each of these cells function, how those functions

267 compare with gene expression in adult M. capitata tissue, and how egg and sperm gene

268 expression is related to each other, it is crucial to statistically test for differential gene

269 expression. As expected, given the divergent different tissue types analyzed, principle

270 components analysis (PCA) shows strong differentiation in gene expression patterns between

271 gametic and adult libraries (Fig. S4).

272 More specifically, with respect to the DEG analysis of egg vs. adult RNA-seq data,

273 DESeq2 identified 13,890 transcripts that were significantly differentially expressed (defined in

274 this study as FDR-adjusted $p$-value $<0.05$ ), of which 4,487 were up-regulated and 9,403 down-

275 regulated. With respect to the differential expression analysis of sperm vs. adult RNA-seq data,

276 DESeq2 identified 9,717 transcripts that were significantly differentially expressed (defined in 
277 this study as FDR-adjusted $p$-value $<0.05$ ), of which 2,985 were up-regulated and 6,732 down-

278 regulated. Log2-fold change estimates (L2FC), all FDR-adjusted $p$-values, putative annotations,

279 blastx hits and percent identity (PID), KO terms, and GO terms are provided for all of these

280 genes the Supplemental spreadsheet (Tabs 10 and 11). The top ten DEGs that had BLAST hits

281 for egg up- and down-regulated and sperm up- and down-regulated are shown in Tables S3 and

282 S4 with all of their differential expression and BLAST statistics and the full lists of DEGS and

283 accompanying annotations are in Supplemental spreadsheet (Tab 3). It is difficult to use these

284 data to gain specific insights because many of the DEGs lack BLAST hits, and those that do,

285 most are to predicted, hypothetical, or uncharacterized proteins in recently sequenced corals.

286 This trend is illustrated in Figure S5 that provides a snapshot of the degree of similarity between

287 the egg and sperm DEG lists as well as the limitations associated with DEGs that have poor 288 annotation.

289 To understand some of the basic functions of the egg and sperm transcriptomes, four sets of DEGs were used as the test sets for separate GO-enrichment analyses (Fisher's Exact Test) against the reference set of all genes in the M. capitata genome (Blast2GO OmicsBox 1.1.164;

292 Götz et al. 2008): (1) egg up-regulated genes (L2FC >1.5; 3,645 DEGs), (2) egg down-regulated genes (L2FC <-1.5; 8,760 DEGs), (3) sperm up-regulated genes (L2FC >1.5; 2,795 genes), and (4) sperm down-regulated genes ( $\mathrm{L} 2 \mathrm{FC}<-1.5 ; 6,606)$. The top enriched $\mathrm{GO}$ terms for egg upregulated genes are "ATP binding”, "phosphatase activity", "positive regulation of biosynthetic process", "protein serine/threonine kinase activity", and "GTP binding". The top enriched GO terms for egg down-regulated genes are "signaling receptor activity", "regulation of transcription by RNA polymerase II", "lipid metabolic process", “organonitrogen compound catabolic process", and "regulation of signaling". The top enriched GO terms for sperm up-regulated genes are "purine nucleotide binding", "purine ribonucleoside triphosphate binding", "enzyme binding", "positive regulation of RNA metabolic process", and "regulation of localization". The top enriched GO terms for sperm down-regulated genes are "positive regulation of cellular metabolic process", "regulation of transcription by RNA polymerase II", "carboxylic acid metabolic process", "regulation of cellular protein metabolic process", and "animal organ development”. The full datasets are represented by the bar charts of combined Biological Process and Molecular Function "most specific" enriched GO terms in Figure S6. 

$>1.5$ ) and down-regulated (L2FC <-1.5) genes (same sets as used above for Fisher's Exact Test) were combined for each of the egg and sperm, indexed with their KO terms retrieved from

310 Kofamscan (Aramaki et al. 2019), and then uploaded into the KEGG "Reconstruct Pathway" tool

311 (Kanehisa 2017). For every pathway, the up- and down-regulated genes were noted and based on

312 trends seen across egg and sperm data in the above enrichment analysis, connections between

313 KEGG pathway activity and known physiological mechanisms of egg and sperm data were

314 made. One major finding from pathway analysis is that nearly every ribosomal protein in both

315 the egg and sperm datasets is down-regulated (Fig. S7). Another intriguing result, when

316 considering DEGs from the egg vs. adult tissue is that "lipid metabolic process" is among the

317 most down-regulated GO terms. Eggs are rich in lipids and lipid activity (Arai et al. 1993;

318 Figueiredo et al. 2012), therefore our data appeared to be anomalous, or resulting from

319 annotation bias. However, upon further inspection, we find that nearly every down-regulated

320 gene associated with this GO term is annotated as a lipase. Therefore, activities associated with

321 lipid breakdown are those down-regulated in the egg.

322

323

Sperm vs. egg comparison

324 PCA analysis of egg vs. sperm cDNA libraries does not show as marked a difference, as found

325 between the egg and sperm libraries compared to the adult (PC1 of egg vs. adult shows 95\%

326 variance, PC1 of sperm vs. adult shows $89 \%$ variance, and PC1 of egg vs. sperm shows $47 \%$

327 variance; Fig. S4). Specifically, with respect to the DEG analysis of egg vs. sperm RNA-seq

328 data, these results are more limited in scope. DESeq2 identified 247 transcripts that were

329 significantly differentially expressed (defined in this study as FDR-adjusted $p$-value $<0.05$ ), of

330 which 108 were up-regulated in the egg (down-regulated in the sperm) and 137 were down-

331 regulated in the egg (up-regulated in the sperm). L2FC was $>1.5,<-1.5$ for all transcripts,

332 therefore no further filtering was needed. L2FC values, all FDR-adjusted $p$-values, putative

333 annotations, blastx hits and percent identity (PID), KO terms, and GO terms are provided for all

334 of these genes in the Supplemental spreadsheet (Tab 13). The sets of egg vs. sperm DEGs were

335 too small to do Fisher's Exact Test for GO enrichment and too sparse to fill in KEGG pathways,

336 therefore the most useful annotations for these genes are from BLAST. The top ten DEGs that 
337 had BLAST hits for this analysis are shown in Table 2 and all annotations are listed in the

338 Supplemental spreadsheet (Tab 13).

339

\section{Discussion}

341 On the most general level, egg and sperm of $M$. capitata each differ considerably in their gene

342 expression when compared with RNA-seq data from adult cells but differ much less when

343 compared to each other. The M. capitata genome contains 63,227 genes, of which 20,220

$344(31.98 \%)$ in the egg and 19,140 (30.27\%) in the sperm are expressed, compared to 37,105

$345(58.69 \%)$ in the adult (these are raw counts in the RNA-seq data, prior to TPM normalization).

346 Because gametes are specialized cells with the purpose of uniting and producing an embryo, it is

347 not surprising that their transcriptomes are more streamlined and specialized. This is seen in our

348 data in the down regulation of ribosomal proteins as well as in the raw RNA-seq data via TPM

349 distributions. What is surprising, however, is the degree of similarity between egg and sperm

350 functional capacity. In addition to their mutual down-regulation of transcription and translation,

351 the most highly expressed genes in the egg and sperm datasets share the same core functions at

352 similar expression levels (Table 1 and Fig. 2). Furthermore, when doing a direct comparison of

353 egg and sperm function with differential expression analysis, DEGs were sparse (247 [most lack

354 annotations]) compared to the numbers of DEGs found in the egg vs. adult and sperm vs. adult

355 datasets $(13,890$ and 9,717 respectively) demonstrating that the degree to which the two gametes

356 differ with respect to function is small.

357

\section{Translation}

359 One of the starkest differences to emerge from the DEG analysis is the marked down-regulation

360 of nearly every ribosomal protein-encoding gene in both the egg and sperm datasets when

361 compared with the adult (Fig. S7). This trend has been documented in human and mammalian

362 systems where sperm are "translationally silent"; i.e., cytoplasmic ribosomal assembly and thus

363 activity is not fully functional when the sperm reaches maturity and nuclear-encoded transcripts

364 may be primarily translated on mitochondrial ribosomes (Gur and Breitbart 2006; Zhao et al.

365 2009; Amaral et al. 2014). This trend has also been documented in the non-coral egg literature as

366 well with studies of Xenopus eggs (Arne et al. 2014) and in mice oocytes, where ribosomal

367 protein expression is repressed during late stage oocyte development (Taylor and Pikó 1992). As 
368 highly specialized cells with much lower overall gene expression compared to adult cells, it is

369

370

371

372

373

374

375

376

377

378

379

380

381

382

383

384

385

386

387

388

389

390

391

392

393

394

395

396

397

398 not surprising for ribosomal protein genes and thus, translation to be down-regulated in both the sperm and egg. However, this topic needs to be further studied to determine whether this phenomenon in sperm is due solely to its role as a gamete or because the cytoplasmic ribosomes may be translationally inactive.

\section{Fertilization}

In broadcast-spawning corals, there are pre-zygotic barriers that gametes must overcome to achieve fertilization (Monteiro et al. 2012, Monteiro et al. 2016), including successful chemical signaling between gametes. Chemical signals are secreted by egg cells to attract sperm. Whether this process is utilized to guide sperm to an egg within the body of an individual organism or the broader environment depends on the system being studied. Regardless, there are two major components to this process: secretion of the chemical attractant by the egg, and taxis initiated by chemical receptors, and made physically possible by motile cilia/ the flagellum of the sperm. In mammalian sperm, for example, motile cilia and the sperm flagellum develop in a similar fashion, have the same axoneme structure, and are virtually identical (Clermont et al. 1993; Avidor-Reiss and Leroux 2015; Wachten et al. 2017). The evolution of compartmentalized sperm cilia (via primary cytosolic ciliogenesis) is necessary for the flagellar movement that propels the sperm cell through its environment to the egg. This is thought to be a universal process in metazoans because it is present in both protostomes (Drosophila) and deuterostomes (mammals, humans), as well as in basal metazoans (corals, sponges) (Avidor-Reiss and Leroux 2015). In broadcast-spawning corals like M. digitata (a relative of $M$. capitata), eggs play a significant role in the regulation of sperm activity and fertilization success, including sperm signaling and stimulation of flagellar motility (Coll et al. 1994; Morita et al. 2006). The signals produced by the egg must be species-specific to prevent hybridization during mass spawning events where many species release gametes into the water column at the same time. In organisms like ascidians and echinoids, $\mathrm{Ca}^{2+}$ has been shown to induce sperm flagellar motility (Yoshida et al. 2003; Morita et al. 2006). A similar effect has been shown experimentally in corals in Acropora species although this alone is insufficient to explain the species-specific nature of sperm and egg union (Morita et al. 2006). It is difficult to find evidence of these complex processes in the summary data of $M$. capitata discussed above due to the large number of genes 
399 lacking annotations. However, upon taking a closer look at the GO data used for Figure 2, for

400 "motile cilium", 79 genes are associated with this GO term, 38 are present in the sperm with

401 TPM counts $>100$, and two of those (g63277 and g9762) are also linked to the GO term "calcium

402 ion binding". Neither of these genes were assigned a KEGG annotation which is why the data

403 for flagellar and motile cilia cellular components are scarce for this analysis. Both of these genes

404 have the same top blastx hit: XP_015777656.1 PREDICTED: uncharacterized protein

405 LOC107355583 isoform X1 in Acropora digitifera. Both of these genes have transcripts that are

406 significantly up-regulated in the sperm vs. adult data and egg vs. adult data. Based on these

407 results, it is possible these genes play a key role in communication associated with the

408 fertilization process. Differential expression and BLAST annotation statistics for these two genes

409 are presented in Figure S8. We speculate that these genes are targets for CRISPR/Cas9 based

410 gene knockdowns (recently developed for Scleractinia; Cleves et al. 2018, 2020) to explore coral

411 reproductive biology.

412

413 Differential expression between egg and sperm

414 As mentioned throughout this study, it is difficult to gain conclusive insights into the functional

415 capabilities of different $M$. capitata cell types due to the lack of annotations for many DEGs. Of

416 the 108 genes significantly up-regulated in the egg when compared to sperm, 26 of these DEGs

$417(\sim 24 \%)$ have top blastx hits to uncharacterized proteins and $12(\sim 11 \%)$ lack an annotation. Of the

418139 significantly up-regulated genes in sperm, $32(\sim 23 \%)$ have top blastx hits to uncharacterized

419 proteins, whereas $13(\sim 9 \%)$ lack an annotation. These results demonstrate that ca. $1 / 3$ of the

420 genes in this dataset, including many of the most highly differentially expressed genes (based on

421 L2FC), do not provide functional information. It is worth noting however, that many of the

422 annotated DEGs in the egg set encode proteins that are localized to the nucleus and take part in

423 mitosis-related or DNA-based processes, whereas the proteins encoded by sperm DEGs often

424 have functions related to motility and ATP-binding. These limited findings are consistent with

425 general gamete physiology, whereby eggs express cell division, transcription, and DNA repair

426 functions and sperm are optimized for energy production to support motility.

427

428 Conclusions 
429 Our findings suggest that coral egg and sperm are not highly differentiated with respect to 430 functional capability. Rather, as motile cells released into the water column and subjected to the 431 same environment prior to fertilization, these morphologically divergent cell types share a 432 conserved gene expression pattern and thus, may be under similar functional constraints. To this 433 end, it would be of interest to investigate egg and sperm transcriptomics in brooding corals (i.e., 434 species that do not release gametes into the water column prior to fertilization) and compare 435 these data to the results of our study.

436 In addition to the major finding of largely shared expression profiles, the data also 437 provide insights into which genes may play key roles in the fertilization process. Due to an 438 association with cell motility and calcium ion binding, two genes (g63277 and g9762) emerge 439 from our dataset as possible candidates for future experimental studies on how eggs and sperm 440 recognize each other in the water column despite the presence of many other gametes from other 441 organisms. We recognize however that our findings are largely summary in nature and serve as 442 an initial step in understanding the transcriptome (vis-à-vis functional capacity) of $M$. capitata 443 gametes. In summary, we present here the first study of the transcriptome of coral sperm and 444 eggs and reach interesting conclusions that pave the way for future multi-omics and genetics 445 investigations on this topic (Cleves et al. 2020), particularly in the context of anthropogenic 446 climate change influences on the marine environment.

447

\section{Acknowledgements}

449 This work was supported by grants from the US National Science Foundation (NSF OCE IEP 4501756616 to DB and NSF OCE, IEP, EPSCoR to HMP), the Israeli Binational Science 451 Foundation (BSF 2016321 to HMP and TM), and the Paul G. Allen Philanthropies. DB was 452 supported by a NIFA-USDA Hatch grant (NJ01170). We would also like to acknowledge Prof. 453 JunMo Lee and Ehud ZelZion for their guidance with various bioinformatics programs.

\section{References}

456 Arai I, Kato M, Heyward A, Ikeda Y, Iizuka T, Maruyama T. 1993. Lipid composition of 457 positively buoyant eggs of reef building corals. Coral Reefs 12:71-75. 
458 Amaral A, Castillo J, Ramalho-Santos J, Oliva R. 2014. The combined human sperm proteome:

459

460

461

462

463

464

465

466

467

468

469

470

471

472

473

474

475

476

477

478

479

480

481

482

483

484

485

486

487

488 cellular pathways and implications for basic and clinical science. Human Reproduction Update 20:40-62.

Aramaki T, Blanc-Mathieu R, Endo H, Ohkubo K, Kanehisa M, Goto S, Ogata H. 2019. KofamKOALA: KEGG ortholog assignment based on profile HMM and adaptive score threshold. Bioinformatics Nov 19, pii: btz859.

Avidor-Reiss T, Leroux MR. 2015. Shared and distinct mechanisms of compartmentalized and cytosolic ciliogenesis. Current Biology 25:R1143-1150.

Baird AH, Bhagooli R, Ralph PJ, Takahashi S. 2009. Coral bleaching: the role of the host. Trends in Ecology and Evolution 24:16-20.

Chiu Y-L, Shikina S, Yoshioka Y, Shinzato C, Chang C-F. 2020. The First De Novo Transcriptome Assembly From the Gonads of a Scleractinian Coral Euphyllia Ancora: Molecular Mechanisms Underlying Scleractinian Gametogenesis. In Review. DOI: 10.21203/rs.3.rs-33092/v1.

Clermont Y, Oko R, Hermo L. 1993. Cell biology of mammalian spermiogenesis. Cell and Molecular Biology of the Testis 1:332-376.

Cleves PA, Strader ME, Bay LK, Pringle JR, Matz MV. 2018. CRISPR/Cas9-mediated genome editing in a reef-building coral. Proceedings of the National Academy of Sciences USA 115:5235-5240.

Cleves PA, Shumaker A, Lee J, Putnam HM, Bhattacharya D. 2020. Unknown to known: advancing knowledge of coral gene function. Trends in Genetics 36:93-104.

Coll JC, Bowden BF, Meehan GV, Konig GM, Carroll AR, Tapiolas DM, Aliño PM, Heaton A, De Nys R, Leone PA, Maida M, Aceret TL, Willis RH, Babcock RC, Willis BL, Florian Z, Clayton MN, Miller RL. 1994. Chemical aspects of mass spawning in corals. I. Spermattractant molecules in the eggs of the scleractinian coral Montipora digitata. Marine Biology 118:177-182.

Concepcion, G.T., Baums, I.B. and Toonen, R.J. 2014. Regional population structure of Montipora capitata across the Hawaiian Archipelago. Bulletin of Marine Science, 90:257275.

Cox EF. 2007. Continuation of sexual reproduction in Montipora capitata following bleaching. Coral Reefs 26:721-724. 
489 Figueiredo J, Baird AH, Cohen MF, Flot J-F, Kamiki T, Meziane T, Tsuchiya M, Yamasaki H.

490

491

492

493

494

495

496

497

498

499

500

501

502

503

504

505

506

507

508

509

510

511

512

513

514

515

516

517
2012. Ontogenetic change in the lipid and fatty acid composition of scleractinian coral larvae. Coral Reefs 31:613-619.

Gibbin, E.M., Putnam, H.M., Gates, R.D., Nitschke, M.R. and Davy, S.K. 2015. Species-specific differences in thermal tolerance may define susceptibility to intracellular acidosis in reef corals. Marine Biology, 162:717-723.

Götz S, García-Gómez JM, Terol J, Williams TD, Nagaraj SH, Nueda MJ, Robles M, Talón M, Dopazo J, Conesa A. 2008. High-throughput functional annotation and data mining with the Blast2GO suite. Nucleic Acids Research 36:3420-3435.

Grottoli AG, Rodrigues LJ, Palardy JE. 2006. Heterotrophic plasticity and resilience in bleached corals. Nature 440:1186-1189.

Gur Y, Breitbart H. 2006. Mammalian sperm translate nuclear-encoded proteins by mitochondrial-type ribosomes. Genes and Development 20:411-416.

Hagedorn M, Carter VL, Lager C, Camperio Ciani JF, Dygert AN, Schleiger RD, Henley EM. 2016. Potential bleaching effects on coral reproduction. Reproduction, Fertility and Development 28:1061.

Hughes TP, Barnes ML, Bellwood DR, Cinner JE, Cumming GS, Jackson JBC, Kleypas J, van de Leemput IA, Lough JM, Morrison TH, Palumbi SR, van Nes EH, Scheffer M. 2017. Coral reefs in the Anthropocene. Nature 546:82-90.

Kanehisa M. 2017. Enzyme Annotation and Metabolic Reconstruction Using KEGG. Methods in Molecular Biology 1611:135-145.

Levitan D, Boudreau W, Jara J, Knowlton N. 2014. Long-term reduced spawning in Orbicella coral species due to temperature stress. Marine Ecology Progress Series 515:1-10.

Love MI, Huber W, Anders S. 2014. Moderated estimation of fold change and dispersion for RNA-seq data with DESeq2. Genome Biology 15:550.

Mass T, Putnam HM, Drake JL, Zelzion E, Gates RD, Bhattacharya D, Falkowski PG. 2016. Temporal and spatial expression patterns of biomineralization proteins during early development in the stony coral Pocillopora damicornis. Proceedings of the Royal Society B: Biological Sciences 283.1829: 20160322.

Peer) reviewing PDF | (2020:03:47283:1:2:CHECK 30 Jun 2020) 
518 Monteiro CA, Paulino C, Jacinto R, Serrão EA, Pearson GA. 2016. Temporal windows of

519

520

521

522

523

524

525

526

527

528

529

530

531

532

533

534

535

536

537

538

539

540

541

542

543

544

545

546 547

reproductive opportunity reinforce species barriers in a marine broadcast spawning assemblage. Scientific Reports 6:29198.

Monteiro CA, Serrão EA, Pearson GA. 2012. Prezygotic barriers to hybridization in marine broadcast spawners: reproductive timing and mating system variation. PLOS ONE 7:e35978.

Morita M, Nishikawa A, Nakajima A, Iguchi A, Sakai K, Takemura A, Okuno M. 2006. Eggs regulate sperm flagellar motility initiation, chemotaxis and inhibition in the coral Acropora digitifera, A. gemmifera and A. tenuis. Journal of Experimental Biology 209:4574.

Moriya Y, Itoh M, Okuda S, Yoshizawa AC, Kanehisa M. 2007. KAAS: an automatic genome annotation and pathway reconstruction server. Nucleic Acids Research 35:W182-185.

Padilla-Gamiño JL, Gates RD. 2012. Spawning dynamics in the Hawaiian reef-building coral Montipora capitata. Marine Ecology Progress Series 449:145-160.

Padilla-Gamiño JL, Weatherby TM, Waller RG, Gates RD. 2011. Formation and structural organization of the egg-sperm bundle of the scleractinian coral Montipora capitata. Coral Reefs 30:371-380.

Putnam HM, Adams DK, Zelzion E, Wagner NE, Qiu H, Mass T, Falkowski PG, Gates RD, Bhattacharya D. 2017. Divergent evolutionary histories of DNA markers in a Hawaiian population of the coral Montipora capitata. PeerJ 5:e3319.

RStudio Team. 2018. RStudio: Integrated Development for R. RStudio, Inc., Boston, MAURL. Available at http://www.rstudio.com/.

Shumaker A, Putnam HM, Qiu H, Price DC, Zelzion E, Harel A, Wagner NE, Gates RD, Yoon HS, Bhattacharya D. 2019. Genome analysis of the rice coral Montipora capitata. Scientific Reports 9:2571.

Smits AH, Lindeboom RG, Perino M, van Heeringen SJ, Veenstra GJ, Vermeulen M. 2014. Global absolute quantification reveals tight regulation of protein expression in single Xenopus eggs. Nucleic Acids Research 42:9880-9891.

Taylor KD, Pikó L. 1992. Expression of ribosomal protein genes in mouse oocytes and early embryos. Molecular Reproduction and Development 31:182-188.

Peer) reviewing PDF | (2020:03:47283:1:2:CHECK 30 Jun 2020) 
548 Wachten D, Jikeli JF, Kaupp UB. 2017. Sperm sensory signaling. Cold Spring Harbor $549 \quad$ Perspectives on Biology 9, pii: a028225.

550 Yoshida, Manabu, Makiko Ishikawa, Hiroko Izumi, Rosaria De Santis, and Masaaki Morisawa.

551 Store-operated calcium channel regulates the chemotactic behavior of ascidian

552 sperm. Proceedings of the National Academy of Sciences 100, no. 1 (2003): 149-154.

553 Zhao C, Guo XJ, Shi ZH, Wang FQ, Huang XY, Huo R, Zhu H, Wang XR, Liu JY, Zhou ZM, 554 Sha JH. 2009. Role of translation by mitochondrial-type ribosomes during sperm capacitation: an analysis based on a proteomic approach. Proteomics 9:1385-1399. 


\section{Figure 1}

Figure 1. Distribution of gene expression in egg and sperm.

Chart showing cellular locations and accompanying GO terms (color-coded to Figure 2) comparing (a) egg and (b) sperm data with respect to the proportion of the respective "expressed" datasets (TPM >100) that are ascribed to each cellular component. This chart is not an exhaustive list of all cellular components (see Supplementary Spreadsheet Tabs 4 and 6 for the full list) but highlights prominent features. Image created with Biorender.com. 
A Egg Expression
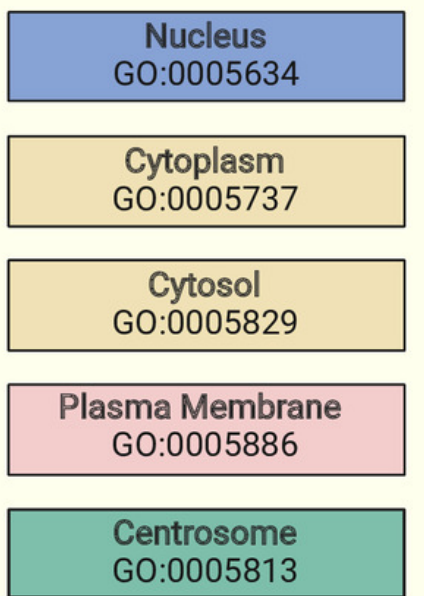

\begin{tabular}{|c|}
\hline Cytoskeleton \\
G0:0005856/0030036 \\
\hline
\end{tabular}

\begin{tabular}{|c|}
\hline Endoplasmic Reticulum \\
G0:0005783 \\
\hline
\end{tabular}
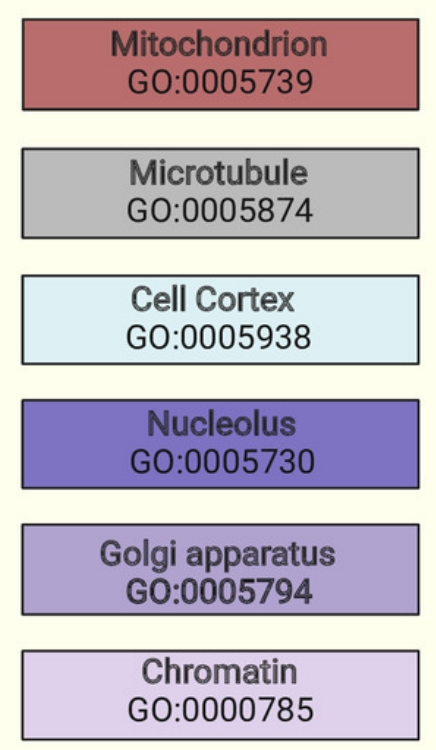

\begin{tabular}{|c|}
\hline Ribosome \\
GO:0022625 \\
\hline
\end{tabular}
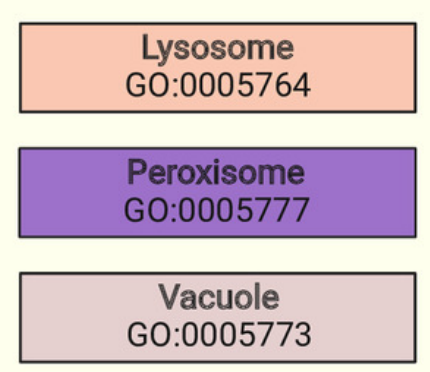

$0.52 \%$

$0.47 \%$

$0.28 \%$

$0.21 \%$

$0.17 \%$

$0.12 \%$

$12.30 \%$

\section{$9.48 \%$}

$5.07 \%$

$2.46 \%$

$2.27 \%$

$1.31 \%$

$1.22 \%$

$1.21 \%$

$0.62 \%$

$0.60 \%$

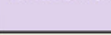

$0.17 \%$

$0.01 \%$
B Sperm Expression

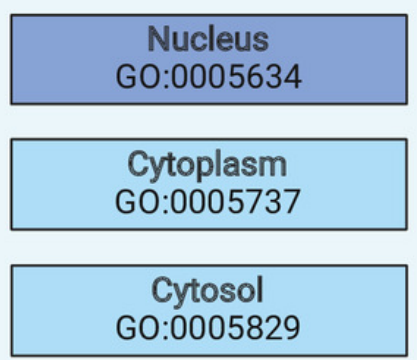

$10.82 \%$

$8.74 \%$

$5.19 \%$

\begin{tabular}{|c|c|}
\hline $\begin{array}{c}\text { Plasma Membrane } \\
\text { G0:0005886 }\end{array}$ & $2.42 \%$ \\
\hline $\begin{array}{l}\text { Centrosome } \\
\text { GO:0005813 }\end{array}$ & $1.70 \%$ \\
\hline $\begin{array}{c}\text { Cytoskeleton } \\
\text { G0:0005856/0030036 }\end{array}$ & $1.61 \%$ \\
\hline
\end{tabular}
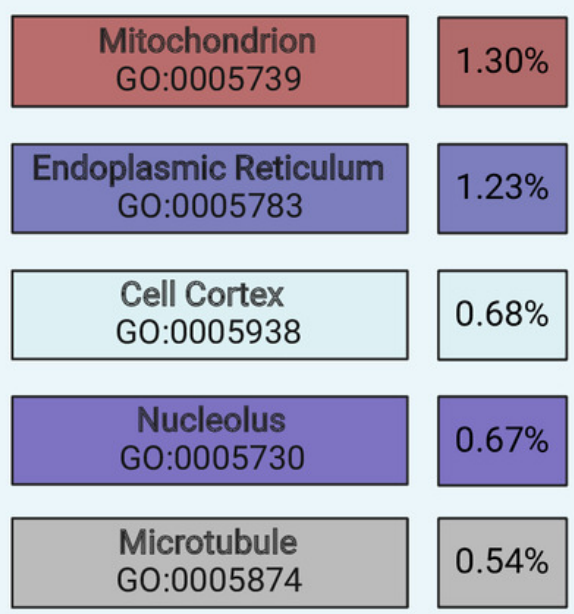

$0.67 \%$

$0.54 \%$

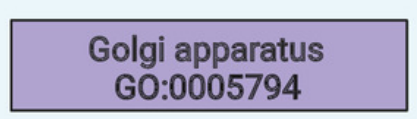

$0.48 \%$

\begin{tabular}{|c|c|}
\hline Ribosome \\
GO:0022625
\end{tabular}

\begin{tabular}{|c|}
\hline Chromatin \\
GO:0000785
\end{tabular}

\begin{tabular}{|c|c|}
\hline $\begin{array}{c}\text { Lysosome } \\
\text { GO:0005764 }\end{array}$ & $0.17 \%$ \\
\hline
\end{tabular}

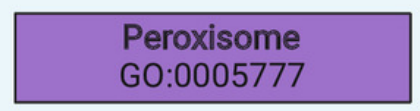

$0.13 \%$

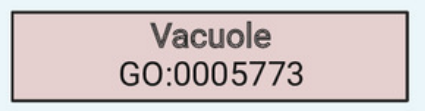

$0.01 \%$ 


\section{Figure 2}

Figure 2. Egg and sperm overall functions.

(A) Schematic image of an M. capitata egg cell depicting cellular structures and organelles associated with GO terms and their accompanying KEGG pathways determined from KO terms. (B) Schematic image of an M. capitata sperm cell depicting cellular structures and organelles associated with $\mathrm{GO}$ terms and their accompanying KEGG pathways determined from KO terms. Image created with Biorender.com. 
A
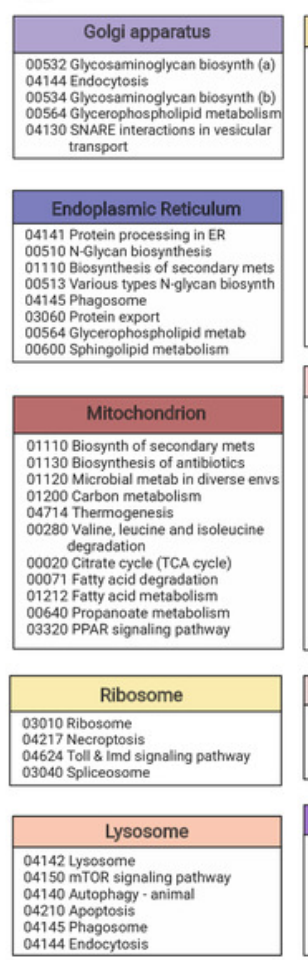

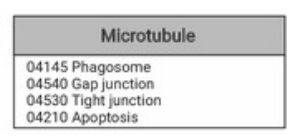

04530 Tight junction
04210 Apoptosis

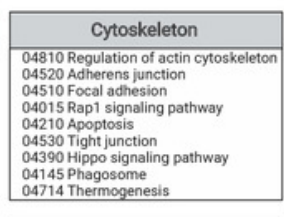

\begin{tabular}{|c|}
\hline Cell Cortex \\
\hline 04015 ap 1 signaling pathway
\end{tabular} \begin{tabular}{|l|}
\hline 04015 Rap1 signaling pathway \\
04810 Reg of actin cytoskeleton
\end{tabular} 04530 Tight junction
04510 Focal adhesion

045144 Encal adhesion
$0410 y+o s i s$ 04011 MAPK signaling pathway 04520 Adherens junction

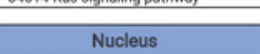

\section{Nucleus}

04110 Cell cycle
04218 Cellular senescence

04114 Oocye meiosis
04113 Meiosis - yeast

03013 RNA transport
03015 mRNA surveillance pathway

03015 mRNA surveillance pathway
04151 P13K.Akt signaling pathway
03050 Proteasome

03050 Proteasome
04120 Ubiquitin mediated proteolysis 04010 MAPK signaling patthway 04010 MAPK signaling pathway
04390 Hippo signaling pathway
04914 Progesterone-mediated ooche 04914 Progesterone
maturation

maturation
03018 RNA degradation
04211 Longevity ragulating pathway 04140 Autophagy - a

030140 Spliceosome
04141 Protein processing in ER

04310 Wnt signaling pathway

03410 Base excision repaí
03030 DNA replication

03030 DNA replication
04350 TGF-beta signaling pathway

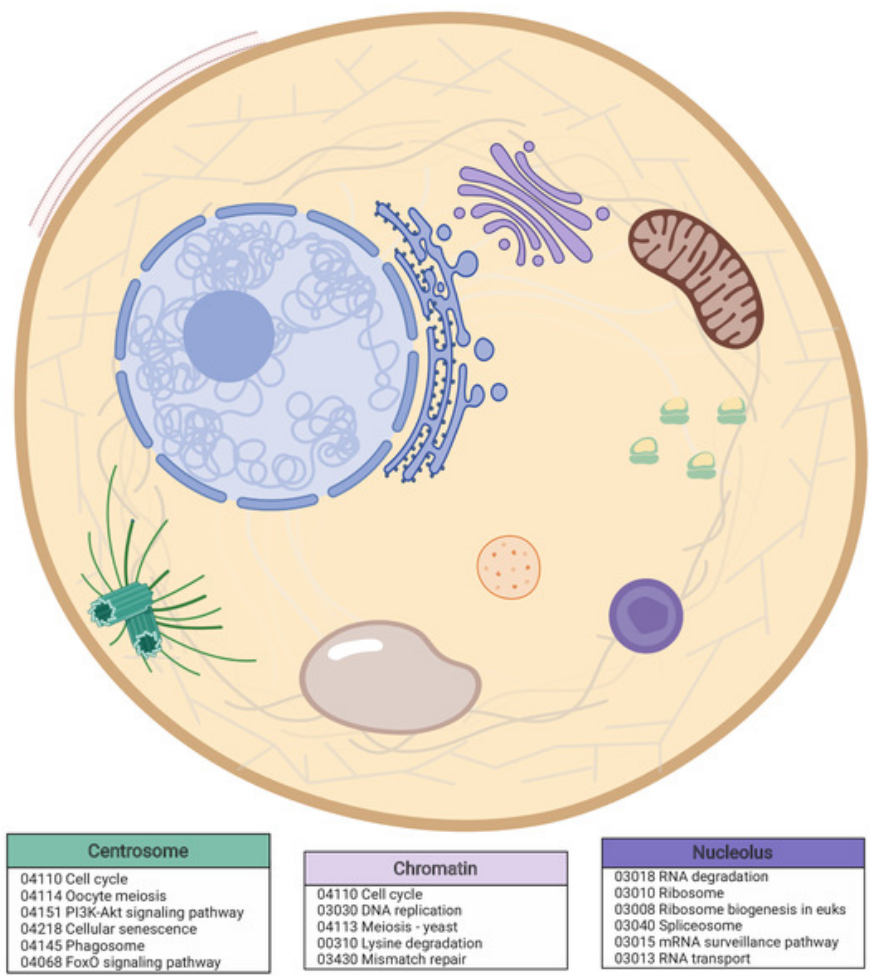

\section{B}

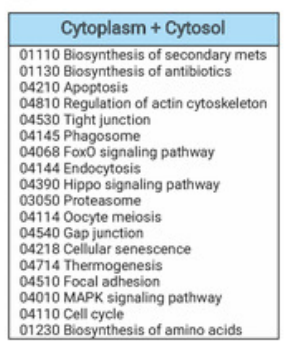

1130 Biogn metabolis 01120 Microbial metab in diverse env 0.0630 Glyoxylate 8 dicarboxylate meto
04213 Longevity regulating pattwway
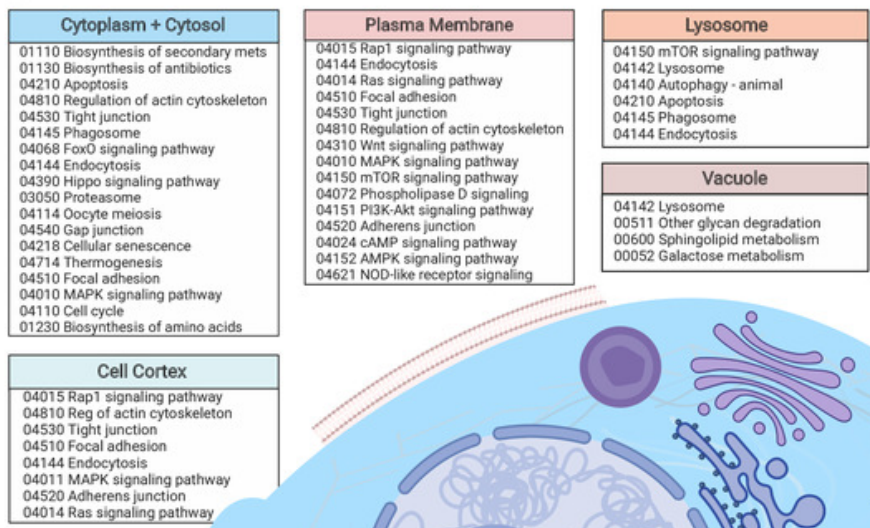

DoO52 Galactose metabolism
Dom

041521 NOD -like receptor signaling
046.
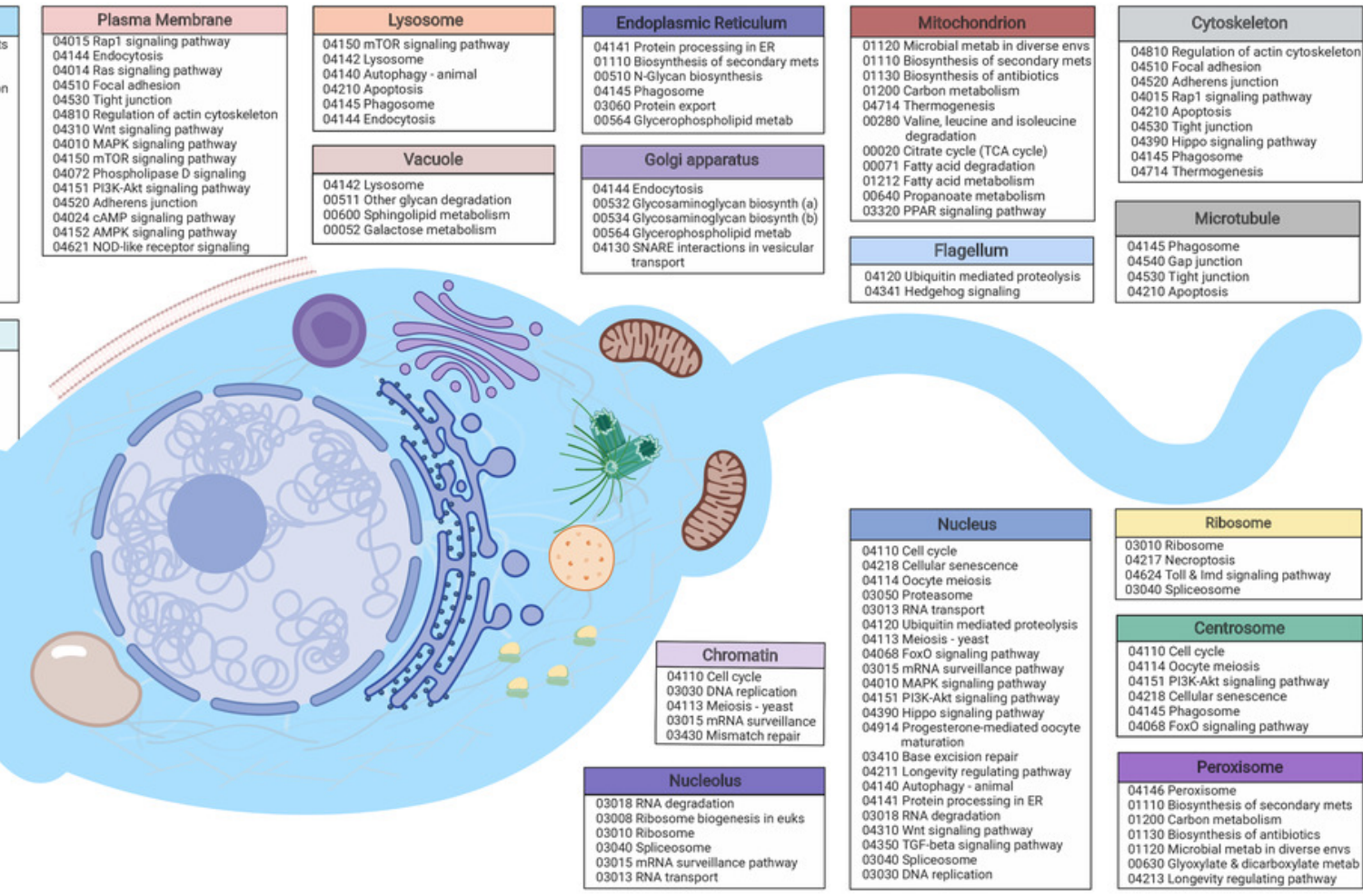

Centrosome

04110 Cell cycle

04114 Oocyte meiosis
04151 P13K-Akt signaling pathway

04218 Cellilar senescence

04068 Foxo signaling pathway

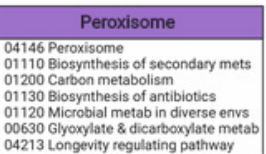




\section{Table $\mathbf{1}$ (on next page)}

Enriched egg and sperm GO terms.

List of enriched GO terms found in the egg, sperm, and egg and sperm "expressed" gene sets. 
1 Table 1. List of enriched GO terms found in the egg, sperm, and egg and sperm "expressed" 2 gene sets.

\begin{tabular}{|c|c|c|}
\hline $\begin{array}{l}\text { Egg "expressed" enriched GO } \\
\text { terms }\end{array}$ & $\begin{array}{l}\text { Enriched GO terms shared by } \\
\text { egg and sperm "expressed" gene } \\
\text { sets }\end{array}$ & $\begin{array}{l}\text { Sperm "expressed" } \\
\text { enriched GO terms }\end{array}$ \\
\hline $\begin{array}{l}\text { Guanyl-nucleotide exchange factor } \\
\text { activity }\end{array}$ & ATP binding & Microtubule binding \\
\hline Phosphoric diester hydrolase activity & GTP binding & Endonuclease activity \\
\hline Meiotic cell cycle & GTPase activity & $\begin{array}{l}\text { Ubiquitin protein ligase } \\
\text { binding }\end{array}$ \\
\hline Cysteine-type endopeptidase activity & Structural constituent of ribosome & Translational elongation \\
\hline Protein processing & Chromatin binding & SNARE binding \\
\hline \multirow{17}{*}{$\begin{array}{l}\text { DNA-templated transcription, } \\
\text { elongation }\end{array}$} & Microtubule motor activity & Protein secretion \\
\hline & Protein polyubiquitination & $\begin{array}{l}\text { Protein localization to } \\
\text { plasma membrane }\end{array}$ \\
\hline & Translation initiation factor activity & RNA helicase activity \\
\hline & Ubiquitin protein ligase activity & $\begin{array}{l}\text { RNA methyltransferase } \\
\text { activity }\end{array}$ \\
\hline & $\begin{array}{l}\text { Negative regulation of transcription } \\
\text { by RNA polymerase II }\end{array}$ & \\
\hline & $\begin{array}{l}\text { Thiol-dependent ubiquitin-specific } \\
\text { protease activity }\end{array}$ & \\
\hline & Rab protein signal transduction & \\
\hline & Unfolded protein binding & \\
\hline & Single-stranded DNA binding & \\
\hline & Actin filament binding & \\
\hline & Magnesium ion binding & \\
\hline & Protein heterodimerization activity & \\
\hline & GTPase activator activity & \\
\hline & Rab GTPase binding & \\
\hline & Peptidyl-serine phosphorylation & \\
\hline & RNA helicase activity & \\
\hline & NAD binding & \\
\hline
\end{tabular}




\section{Table 2 (on next page)}

Annotation of top DEGs in egg and sperm comparisons.

Annotation of the top ten DEGs with BLAST hits in the comparison of egg and sperm RNA-seq libraries. PID is protein identity. 
1 Table 2. Annotation of the top ten DEGs with BLAST hits in the comparison of egg and sperm

2 RNA-seq libraries. PID is protein identity.

3 Up-regulated in egg/down-regulated in sperm

\begin{tabular}{|l|l|l|l|l|}
\hline Gene Name & $\begin{array}{l}\text { Log2 fold } \\
\text { change }\end{array}$ & p-adjusted & BLASTx Annotation & PID \\
\hline adi2mcaRNA25316_R8 & 7.71554159 & $2.15 \mathrm{E}-11$ & $\begin{array}{l}\text { >XP_022808494.1 histone H2B-like } \\
\text { [Stylophora pistillata] }\end{array}$ & 92.3 \\
\hline adi2mcaRNA9523_R7 & 7.69856143 & $1.36 \mathrm{E}-05$ & $\begin{array}{l}\text { >PFX24216.1 E3 ubiquitin-protein } \\
\text { ligase TRIM71 [Stylophora } \\
\text { pistillata }]\end{array}$ & 49.2 \\
\hline g21152 & 7.4243888 & $3.69 \mathrm{E}-05$ & $\begin{array}{l}\text { >XP_015772650.1 PREDICTED: } \\
\text { tyrosine-protein kinase Fer-like } \\
\text { [Acropora digitifera] }\end{array}$ & 79.4 \\
\hline adi2mcaRNA9523_R9 & 7.25684272 & $5.25 \mathrm{E}-05$ & $\begin{array}{l}\text { >PFX24216.1 E3 ubiquitin-protein } \\
\text { ligase TRIM71 [Stylophora } \\
\text { pistillata] }\end{array}$ & 49.5 \\
\hline g12388 & 7.18366792 & $7.45 \mathrm{E}-05$ & $\begin{array}{l}\text { >XP_020610552.1 protein Mpv17- } \\
\text { like [Orbicella faveolata] }\end{array}$ & 85.9 \\
\hline g6237 & 6.85148779 & $3.02 \mathrm{E}-04$ & $\begin{array}{l}\text { >PFX17025.1 Poly [ADP-ribose] } \\
\text { polymerase 14 [Stylophora pistillata] }]\end{array}$ & 54.3 \\
\hline adi2mcaRNA18203_R4 & 6.40487831 & $2.14 \mathrm{E}-03$ & $\begin{array}{l}\text { >PFX19948.1 Coiled-coil domain- } \\
\text { containing protein 150 [Stylophora } \\
\text { pistillata] }\end{array}$ & 70.8 \\
\hline g48873 & 6.2654406 & $6.74 \mathrm{E}-03$ & $\begin{array}{l}\text { >XP_020623935.1 centromere } \\
\text { protein N-like isoform X1 [Orbicella } \\
\text { faveolata] }\end{array}$ & 61.6 \\
\hline g18750 & 6.17777817 & $4.36 \mathrm{E}-03$ & $\begin{array}{l}\text { >PFX27818.1 Amiloride-sensitive } \\
\text { amine oxidase [copper-containing] } \\
\text { [Stylophora pistillata] }\end{array}$ & 63.2 \\
\hline
\end{tabular}

Up-regulated in sperm/down-regulated in egg

\begin{tabular}{|l|l|l|l|l|}
\hline $\mathrm{g} 9290$ & 11.493954 & $1.24 \mathrm{E}-03$ & $\begin{array}{l}\text { >XP_015776821.1 PREDICTED: } \\
\text { creatine kinase B-type-like isoform } \\
\text { X1 [Acropora digitifera] }\end{array}$ & 83.9 \\
\hline $\mathrm{g} 34129$ & 9.4025289 & $1.23 \mathrm{E}-06$ & $\begin{array}{l}\text { >XP_015757707.1 PREDICTED: } \\
\text { fibropellin-1-like isoform X3 } \\
\text { [Acropora digitifera }]\end{array}$ & 50.5 \\
\hline $\mathrm{g} 22489$ & 8.8676566 & $3.42 \mathrm{E}-06$ & $\begin{array}{l}\text { >XP_015774900.1 PREDICTED: } \\
\text { cofilin-like isoform X1 [Acropora } \\
\text { digitifera }]\end{array}$ & 79.2 \\
\hline $\mathrm{g} 24088$ & 8.4749941 & $7.84 \mathrm{E}-11$ & $\begin{array}{l}\text { >XP_020610152.1 ADP-ATP carrier } \\
\text { protein 3, mitochondrial-like } \\
\text { [Orbicella faveolata }]\end{array}$ & 87.4 \\
\hline $\mathrm{g} 43213$ & 8.228315 & $4.44 \mathrm{E}-05$ & $\begin{array}{l}\text { >XP_020620813.1 UPF0573 protein } \\
\text { C2orf70 homolog B-like [Orbicella } \\
\text { faveolata] }\end{array}$ & 63.8 \\
\hline
\end{tabular}




\begin{tabular}{|l|l|l|l|l|}
\hline $\mathrm{g} 1536$ & 8.0816351 & $4.44 \mathrm{E}-05$ & $\begin{array}{l}\text { >XP_020600610.1 lymphocyte } \\
\text { antigen 6H-like isoform X2 } \\
\text { [Orbicella faveolata }]\end{array}$ & 48.5 \\
\hline $\mathrm{g} 50437$ & 7.8691611 & $4.02 \mathrm{E}-04$ & $\begin{array}{l}\text { >XP_015777745.1 PREDICTED: } \\
\text { agrin-like [Acropora digitifera] }\end{array}$ & 50.5 \\
\hline $\mathrm{g} 24451$ & 7.7600705 & $7.53 \mathrm{E}-05$ & $\begin{array}{l}\text { >XP_020615035.1 MORN repeat- } \\
\text { containing protein 5-like [Orbicella } \\
\text { faveolata] }\end{array}$ & 55.8 \\
\hline $\mathrm{g} 61108$ & 7.5970964 & $3.11 \mathrm{E}-03$ & $\begin{array}{l}\text { >XP_015777385.1 PREDICTED: } \\
\text { stabilizer of axonemal microtubules } \\
\text { 2-like isoform X2 [Acropora } \\
\text { digitifera] }\end{array}$ & 80.4 \\
\hline $\mathrm{g} 48486$ & 7.5070947 & $5.19 \mathrm{E}-03$ & $\begin{array}{l}\text { >XP_015756818.1 PREDICTED: } \\
\text { mitochondrial glutamate carrier 2- } \\
\text { like [Acropora digitifera] }\end{array}$ & 86.2 \\
\hline
\end{tabular}

4 